\title{
Computational Framework to Support Development of Applications Running on Multiple Co-located Devices
}

\author{
Pedro Albuquerque Santos \\ NOVA LINCS, NOVA University of Lisbon \\ Portugal \\ Sustain.RD, ESTSetúbal, Instituto Politécnico de Setúbal \\ Portugal \\ pe.santos@campus.fct.unl.pt \\ Rui Porfírio \\ NOVA LINCS, NOVA University of Lisbon \\ Portugal \\ rui.porfirio@campus.fct.unl.pt
}

\author{
Rui Neves Madeira \\ NOVA LINCS, NOVA University of Lisbon \\ Portugal \\ Sustain.RD, ESTSetúbal, Instituto Politécnico de Setúbal \\ Portugal \\ rui.madeira@estsetubal.ips.pt \\ Nuno Correia \\ NOVA LINCS, NOVA University of Lisbon \\ Portugal \\ nmc@fct.unl.pt
}

\begin{abstract}
We designed a framework to generalize the development of applications with UI elements distributed across co-located devices. The framework is comprised of diverse components in order to deal with the complexity of such a task, including: authentication and authorization services; a broker to sync information across multiple application instances; background services that gather the capabilities of the devices; an indoor positioning system to determine when devices are close to each other; and a library which helps integrating Web applications with the broker, determining which components to show based on UI requirements and device capabilities, and custom elements to manage the distribution of the UI components and the multiple UI application states. Collaboration is supported by sharing UI states with other users.
\end{abstract}

\section{CCS CONCEPTS}

- Human-centered computing $\rightarrow$ Human computer interaction (HCI); Ubiquitous and mobile computing; • Computer systems organization $\rightarrow$ Distributed architectures; $\bullet$ Software and its engineering $\rightarrow$ Software libraries and repositories; Development frameworks and environments.

\section{KEYWORDS}

multi-device applications, cross-device interaction, context-awareness, application framework, proxemics, indoor positioning, UX, HCI

ACM Reference Format:

Pedro Albuquerque Santos, Rui Neves Madeira, Rui Porfírio, and Nuno Correia. 2021. Computational Framework to Support Development of Applications Running on Multiple Co-located Devices. In Companion of the 2021 ACM SIGCHI Symposium on Engineering Interactive Computing Systems

This work is licensed under a Creative Commons Attribution-Share Alike International 4.0 License.

EICS '21 Companion, June 8-11, 2021, Virtual Event, Netherlands

(c) 2021 Copyright held by the owner/author(s).

ACM ISBN 978-1-4503-8449-0/21/06

https://doi.org/10.1145/3459926.3464758
(EICS '21 Companion), fune 8-11, 2021, Virtual Event, Netherlands. ACM, New York, NY, USA, 7 pages. https://doi.org/10.1145/3459926.3464758

\section{INTRODUCTION}

People use an increasingly large number of computing devices in their daily lives [15]. However, applications are usually confined to run on a single device at any given time. At best, they are capable of synchronizing certain types of data, and such synchronization often needs to be explicitly turned on $[5,8,33]$.

Therefore, there are missed opportunities to build integrated experiences that run across multiple devices. We believe that it is important and beneficial to take advantage of the devices around us by combining them and integrating their resources. It should be possible to leverage the strengths of some devices in certain scenarios, while minimizing the weaknesses of other devices in some situations.

This realization motivated us to develop the tools needed to build applications that have their user interface (UI) seamlessly distributed across multiple co-located devices. In order to fulfill our vision, we designed a framework that generalizes the development of this type of applications and that allows third-party developers to take advantage of the increasing pervasiveness of digital devices.

The initial version of the framework laid the foundation needed to build cross-device application experiences [31]. Later, we focused on the issue of automatically distributing UI components among co-located devices by matching the description of the capabilities of each of the co-located devices and the requirements of each of the application's components [32].

The current version allows users to switch between automatic and manual distribution of the application's UI elements by device. Moreover, while the framework initially kept the current per user and application's UI state synchronized across the application instances running on multiple devices, the framework has since been extended to allow storing multiple UI states per user and application. Furthermore, these UI states can be shared to enable collaboration between multiple users.

These additions were accompanied by the development of reusable components that can be easily integrated into applications. This should allow developers to quickly give users direct control over 
the distribution of UI components, the management of multiple UI states, and to enable collaboration by sharing those states.

In the remaining of this paper, we introduce the research areas that support our work (section 2). We then present a technical summary of our framework (section 3 ). We finish by presenting conclusions and ideas for future work (section 4).

\section{RELATED WORK}

The framework concept came from previous research on system specific efforts that combined mobile devices with situated displays $[25,30]$. We also realized that people started to spontaneously use multiple screens at the same time, e.g., by using mobile devices while watching TV to access trivia and engage with other viewers $[9,20,21]$. There are already interesting works related to second screen applications that provide an enhanced viewing experience $[3,4]$. Moreover, based on device shipment trends and developer interest, a multi-device application ecosystem has been previously identified as the next way forward [35]. Users should be able to seamlessly move between co-located devices, performing tasks across them, such as continuing playing the same film on another device, or displaying the playback controls on one device and the media output on another one.

Liquid Software is an approach in which applications and data flow seamlessly across devices, allowing users to continuously switch from one device to the next without having to worry about how to transfer applications, settings or associated data [13]. Responsive Web design partially addresses the issue of running software fluidly on different screen sizes and input modalities, but we still have challenges, such as code mobility, state synchronization, UI adaptation and shared data access [19]. Liquid.js is a framework that tackles some of them to enable multi-device applications, which operate on a shared decentralized state using Web-based technologies [7]. XDBrowser is another example which segments Web pages and distributes the fragments across devices [22]. Meanwhile, Vistribute provides a data analysis framework that adapts to a multitude of devices based on information about the views and the data they visualize [14].

There is also research that is focused on the design space of the multi-device applications. For instance, McKit contains scenarios, hint cards, and a framework for understanding the required trade-offs in realistic contexts of use [23], while the $4 C$ framework identifies four principles for multi-device interaction design: communality, collaboration, continuity, and complementarity [34]. Paternò and Santoro present a logical framework for understanding, analyzing, and comparing features of multi-device UIs [28]. More recently, Paternò published an in-depth survey and classification of research in this area [27]. However, despite many progresses, there are still important challenges to overcome [6].

The anthropological concept of proxemics is also important to this research. It identifies the culturally dependent ways in which people use interpersonal distance to understand and mediate their interactions [11]. Likewise, users should expect increasing connectivity and interaction possibilities as they bring their devices closer together [10]. Saul Greenberg has been one of the most significant contributors to this area. His team developed a Proximity Toolkit to test multiple proxemic-aware applications [17] and GroupTogether to enable co-located collaboration across multiple devices by using sensors placed in the devices and in the environment to capture the relationship between users and their devices [18]. They also introduced the Gradual engagement design pattern, which describes how to design interfaces that gradually engage the user by disclosing connectivity and information exchange capabilities as a function of inter-device proximity across three stages [16]. In order to determine the proxemic relationships between multiple devices to enable association and interaction across them, we also developed an indoor positioning system (IPS) along with our framework based on Wi-Fi and Bluetooth Low Energy after carefully studying existing solutions [2].

\section{SOLUTION}

We developed the YanuX framework to support the automated distribution of UI elements across multiple co-located devices. However, users should be able to easily override the choices that were made for them. Besides that, collaboration should also be fostered in an environment where multiple users and devices meet. This section summarizes the technical aspects of the framework.

\subsection{Architecture}

Figure 1 shows a diagram of the framework's architecture. The components at the bottom are server-side components (IPS Server, YanuX Auth, YanuX Broker and YanuX IPS Bridge). The devices that run applications based on the framework appear on top.

Each device can host multiple applications (Apps), which access the framework's services through a library (YanuX Coordinator) that abstracts lower level details and provides tools to deal with component distribution and manage the application state. Application instances may also communicate with the YanuX Broker by establishing a connection using the Feathers Client library or Socket.io. The IPS Client and YanuX Orchestrator run in the background on each device and are shared by all applications.

As for the arrows connecting the components, a single-sided arrow means a request is made from the source component to the target component. Double-sided arrows represent that a bidirectional channel is kept open between the two components once the communication is established from a client component, on one of the devices, to a server component.

More information, documentation and the repositories for all of the components of the framework can be found at https://yanuxframework.github.io/.

\subsection{YanuX Auth}

We need to keep track of the applications, developers and users that are using the YanuX Framework. Therefore, YanuX Auth consists of a website, as well as related authentication and authorization services. It implements an OAuth 2.0 Authorization Server [12] with PKCE (Proof Key for Code Exchange) [1] support that issues access tokens used by the client applications to make requests to other components on behalf of each user. A Resource Server was also implemented to expose a service to check the validity of an access token as well as a standard OpenID Connect endpoint [29]. 


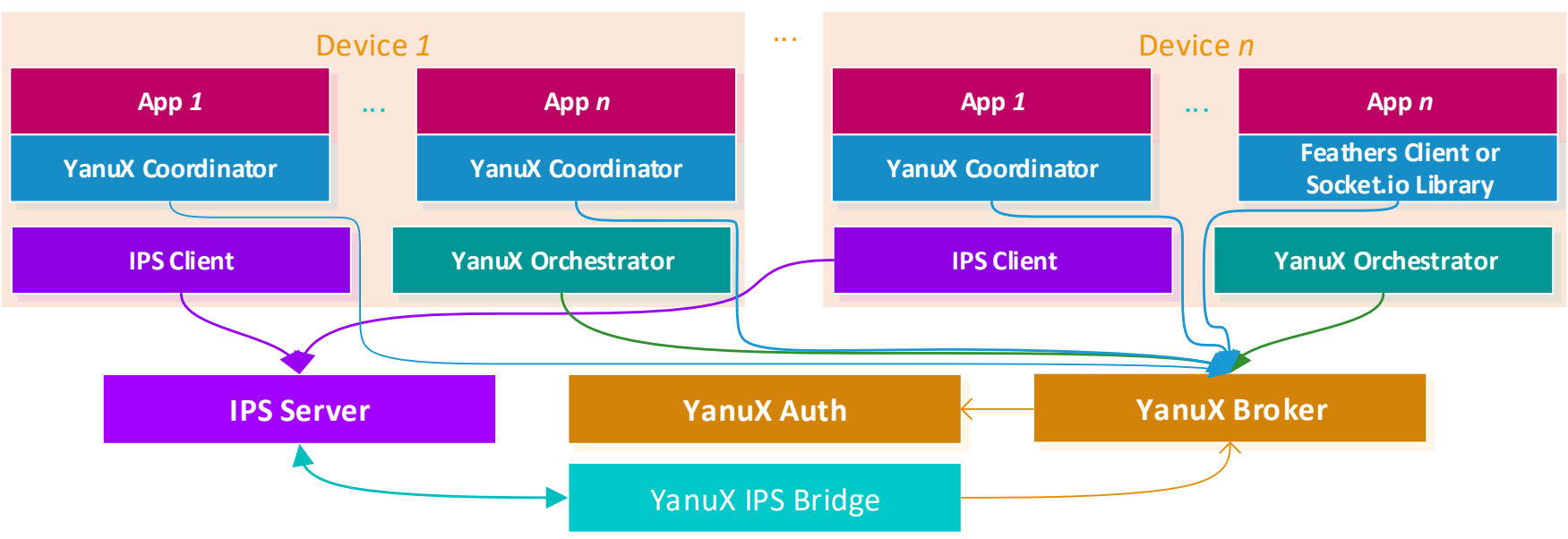

Figure 1: YanuX Framework Simplified Architecture

\subsection{YanuX Broker}

We need to keep track of the UI state across devices. Therefore, the YanuX Broker exposes ISON (JavaScript Object Notation) objects that should be used by applications to store the global UI state. The application instances on each device can then manipulate and subscribe to changes made to those objects. Within the context of our framework, those objects are called resources. Each user can have multiple resources stored for each application. It is also possible to share resources with other users to enable collaboration.

The YanuX Broker supports the submission of events because there are some interactions that do not translate well to direct changes to the global UI state and the re-rendering of client application instances on each of the devices based on the propagation of those changes. Each event should have an associated name, payload (a ISON object) and the identifier of the resource that the user is currently subscribed to. The event is sent to any instances that are subscribed to the same resource.

The YanuX Broker is also responsible for aggregating data coming from the YanuX IPS Bridge into per-user proxemic states and keeping subscribing application instances up-to-date. The proxemic states can be fed to the YanuX Coordinator library to determine which UI elements should be shown. When a user is subscribed to a shared resource its proxemic state is shared with the same users.

The YanuX Broker exposes its services through a REST API: http: $/ /$ j.mp/yanuxbroker. However, the same set of services are also made available to Feathers or Socket.io compatible clients. These allow the server to push events to connected clients, which is essential for interactive applications.

\subsection{YanuX Coordinator}

The services provided by the YanuX Broker can be directly accessed using any of the previously mentioned options. However, we have written in TypeScript a helper library called YanuX Coordinator, which makes it easier to access the YanuX Broker from applications based on Web technologies. It also offers non-trivial functionality that is not be easily re-implemented.
The library takes care of lower-level details so that third-party developers can focus on building their own applications. Developers only need to provide the authentication token, which they have acquired from YanuX Auth, and to register callback functions for the events that the server can push to connected clients. Depending on the type of event, developers may program their applications to simply change a minor part of the UI or completely re-render it.

The API of the YanuX Coordinator can be summarized as follows:

- constructor(brokerUrl, localDeviceUrl, clientId, credentials): The constructor that creates a Coordinator object with the details needed to connect to the YanuX Broker.

- Properties:

- client: Client application that is connected to the broker.

- device: Device running the current application instance.

- instance: Local instance of the application.

- proxemics: Proxemic relationships of the devices the user has access to, i.e., including those that are available via resource sharing with other users.

- resource: Default resource of the user on the current app.

- subscribedResourceId: Id of the subscribed resource.

- user: The user using the current application instance.

- Methods (arguments with ? have a default value):

- createResource(resourceName?): Creates a new resource.

- deleteResource(resourceId): Deletes a resource.

- emitEvent(value, name): Emits an application event.

- getActiveInstances(): Gets active instances that the current user has access to, i.e., including those that are available via resource sharing with other users.

- getInstances(extraConditions?): Gets instances that the current user has access to, i.e., including those that are available via resource sharing with other users.

- getProxemicsState(): Gets the proxemic relationships of the devices the user has access to, i.e., including those that are available via resource sharing with other users.

- getResourceData(resourceId?): Gets a resource's data.

- getResources(): Gets the resources that the user currently has access to, i.e, including shared resources.

- init(): Initializes the connection to the broker. 
- isConnected(): Indicates if the Coordinator is connected.

- logout(): Logs out from the Broker.

- setComponentDistribution(components, auto?, instanceId?): Sets the distribution of UI components.

- setInstanceActiveness(active): Sets if the current instance is active, i.e., if it is being used.

- setResourceData(data, id?): Sets a resource's data.

- shareResource(userEmail, resourceId?): Shares a resource with a user.

- subscribeEvents(subscriberFunction): Subscribe to application events.

- subscribeInstances(subscriberFunction): Subscribe to changes made to instances.

- subscribeProxemics(subscriberFunction): Subscribe to changes made to the proxemics that user has access to, i.e., including those that are available via resource sharing with other users.

- subscribeReconnects(subscriberFunction): Subscribe to reconnection events.

- subscribeResource(subscriberFunction, resourceId?): Subscribe to changes made to a resource.

- subscribeResourceSubscription(subscriberFunction): Subscribe to changes made to the resource subscription, i.e., which resource is subscribed by the user.

- subscribeResources(subscriberFunction): Subscribe to changes to made to the resources that a user has access to, i.e., owned or shared with.

- unshareResource(userEmail, resourceId?): Unshares a resource that was shared with another user.

- unsubscribeEvents(), unsubscribeInstances(), unsubscribeProxemics(), unsubscribeReconnects(), unsubscribeResource(), unsubscribeResourceSubscription(), unsubscribeResources(): Unsubscribe from the corresponding subscriptions mentioned above.

- updateInstanceActiveness(): Determines if the current instance is being used and sets its activeness accordingly.

More details are available here: http://j.mp/yanuxcoordinator.

3.4.1 Resource Management. Since the framework can store multiple application states (resources) per user and application. This can be useful for multi-tasking, e.g., multiple opened documents, seamlessly switching back and forth between multiple videos, or participating in multiple conversations. Resources can also be shared with other users to enable collaboration in the same space. For instance, we can envision collaborative multi-device document editors, classroom applications or digital canvases.

Therefore, we have developed a Web component named YanuX Resource Management Element to assist developers with the management of resources in an application's UI (see Figure 2).

The list of resources owned by or shared with a user are fed into the Web component. They are listed in a drop-down list and an event is triggered when users select a resource so that developers can instruct the YanuX Coordinator to retrieve and subscribe to it.

The buttons below the drop-down list allow users to create, rename, share or delete a resource. There is a second drop-down that will be populated with the e-mail of the users with whom the resource has been shared. The owner is able to click on the button

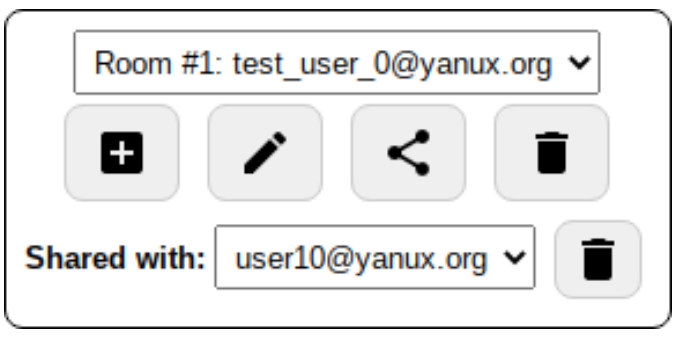

Figure 2: YanuX Resource Management Element.

next to the list to stop sharing the resource with the currently selected user. These actions show a dialog box asking for extra input or confirmation. Once confirmed, the Web component fires an event which let developers know that they should make the relevant YanuX Coordinator API calls to actually perform the operations.

The YanuX Resource Management Element API can be summarized as follows:

- HTML Attributes:

- resources: The resources that the user has access to, , i.e, including shared resources.

- selectedResource: The currently selected resource.

- selectedResourceId: The Id of the selected resource.

- userId: The Id of the current user.

- Custom Events:

- create-resource: The detail property contains the resourceName of the created resource.

- delete-resource: The detail property contains the resourceId of the deleted resource.

- rename-resource-name: The detail property has the renamed resource, its resourceId and its new resourceName.

- resource-selected: The detail property contains the selected resource and its resourceId.

- share-resource-email: The detail property contains the resource, its resourceId and the userEmail of the user with whom the resource was shared.

More details are available here: http://j.mp/yanuxresman.

3.4.2 Component Distribution. Another key aspect of YanuX Coordinator is the automatic distribution of UI components. The YanuX Orchestrator gathers information about each device's capabilities and sends it to the YanuX Broker. Meanwhile, the YanuX Broker keeps track of the connected instances of each application and the YanuX Coordinator is also able to determine if an instance is inactive, i.e., it is running in the background.

Developers need to add an object to their application that follows our $7 S O N$-based DSL (Domain Specific Language), as defined here: http://j.mp/yanuxcompres. The name of the properties of the object are the unique names of the UI components of the application. For each component, the developer must use our DSL to define restrictions and preferences that will be taken into account when determining whether a component should be visible.

The API of our ComponentsRuleEngine can be summarized as follows:

- constructor(localInstanceUuid, localDeviceUuid): Initializes some properties of the distribution engine. 
- Properties:

- currentComponentsDistribution: Current distribution of UI components.

- instances: Instances considered by the algorithm.

- localDeviceUuid: UUID of the local device.

- localInstanceUuid: Id of the local instance.

- proxemics: Proxemic relationships of the devices the user has access to, i.e., including those that are available via resource sharing with other users.

- restrictions: DSL that imposes restrictions on the distribution of components.

- Methods:

- run(ignoreManual?): Runs the distribution algorithm. ignoreManual=true resets the distribution to automatic.

More details are available here: http://j.mp/yanuxcompruleng

The automatic distribution of components strives to come up with the best solution. However, users should be able to override it because they might prefer a different distribution. Therefore, we have created a Web component named YanuX Components Distribution Element that allows developers to give the control over the distribution of UI components back to users (see Figure 3).

\begin{tabular}{|c|c|c|c|c|c|}
\hline \multicolumn{6}{|c|}{$\begin{array}{c}\text { Device: Laptop } \\
\text { Instance: Instance } 1\end{array}$} \\
\hline Device & Video Call & Camera & Chat Messages & Chat Input & Auto \\
\hline $\begin{array}{l}\text { Laptop } \\
\text { (Instance 1) }\end{array}$ & $\nabla$ & $\square$ & $\square$ & $\square$ & ப) \\
\hline $\begin{array}{l}\text { Laptop } \\
\text { (Instance 2) }\end{array}$ & $\square$ & $\square$ & $\nabla$ & $\nabla$ & (1) \\
\hline Tablet & $\square$ & $\nabla$ & $\nabla$ & $\square$ & (1) \\
\hline Smartphone & $\square$ & $\square$ & $\square$ & $\nabla$ & (1) \\
\hline
\end{tabular}

Figure 3: YanuX Components Distribution Element

The names of the device and of the instance where the element is placed are displayed at the top. Figure 3 shows a total of four applications instances running on three distinct devices and four components. The headers of the 2 nd to 5 th columns display the name of the components while the rows below contain check boxes reflecting the distribution of components across the active application instances. Users can use them to choose if a component should be shown on the corresponding application instance listed in the 1 st column. When that happens, the web component emits an event.

The 6th column has buttons that indicate if the distribution for a certain application instance was determined automatically (pressed green state) or if it was set by the user (unpressed red state). If a user changes the distribution of components, the corresponding button will become unpressed and red. Moreover, when a user presses one of those buttons the YanuX Components Distribution Element generates an event signaling that the user wants the distribution of the UI components of the corresponding instance to be automatic.

The YanuX Coordinator's distribution algorithm should be invoked whenever there are changes in the proxemic relationships of co-located devices or in the available instances, when there is a manual change in the distribution of components, or when a users requests to reset the distribution of an instance. The algorithm gives priority to the distribution made by the user.

Otherwise, it will take into account the restrictions defined by the developer, the proxemic relationships between devices, whether each application instance is active, and the capabilities of the devices running those instances. The algorithm runs independently on each application instance and returns a favaScript object with a boolean property for each component that indicates whether it should be shown. It is up to the developer to interpret these values.

The YanuX Components Distribution Element API can be summarized as follows:

\section{- HTML Attributes:}

- componentsDistribution: Distribution of UI components.

- instanceId: The Id of the current instance.

- Custom Events:

- reset-auto-components-distribution: The detail property contains the instanceId that had its distribution reset.

- updated-components-distribution: The detail property contains the instanceId, the name of the component, the checkboxChecked status and the updated componentsDistribution.

More details are available here: http://j.mp/yanuxcompdist

\subsection{Indoor Positioning System (IPS)}

The framework needs to have an updated perspective of the devices available in the surrounding environment. It relies on the awareness of the devices' proxemic relationships, specifically the distance, orientation, and identity dimensions [10]. Since there is no readily available solution that enables us to capture that information, we had to develop our own.

The IPS Client is available for Android and Linux. It scans for BLE (Bluetooth Low Energy) beacons and $W i-F i$ access points, and sends its findings to the IPS Server along with the orientation reported by the device's sensors. The server estimates the device's position and publishes the results to a WAMP (Web Application Messaging Protocol) Router running besides it [37]. Any interested party can subscribe to those position updates.

The IPS Server supports radio map fingerprinting and proximity sensing techniques that apply machine learning algorithms to predict a device's absolute position in an area and the relative position to BLE beacons, respectively. Trilateration is also supported and it applies the method of least squares to estimate a device's position based on the distance to the known location of at least three $B L E$ beacons. Moreover, the server uses fuzzy logic to decide what is the most appropriate positioning technique in a specific situation. By following this procedure, the system's performance is expected to be at least equal to the performance achieved by the best performing algorithm [24].

\subsection{YanuX IPS Bridge}

The YanuXIPS Bridge integrates the framework with the positioning system by subscribing to the IPS Server through the WAMP protocol [37]. It tracks successive events from the same device and passes them through an exponential moving average filter to smooth out 
any errors before sending the measurements to the YanuX Broker for futher processing.

Proxemic relationships are established between devices that belong to users that have access to the currently selected resources (i.e., both owners and users that the resources have been shared with), if their positions are within 3 meters of each other and their orientations deviate by less than 90 degrees. These values were chosen based on empirical experiments that were performed during the development of the solution.

\subsection{YanuX Orchestrator}

The functions may be the same but multi-device applications must run on a broad variety of devices with different input and output modalities [26]. Screens may range from the tiny ones found on smartwatches, to large TV screens, while there are devices that may not have a screen, e.g., a smart speaker. Input may come from a T9 keypad, a touchscreen, a mouse, or a physical QWERTY keyboard.

A single UI solution will most likely not fit the full range of possibilities [36]. The UI of these applications should be responsive and adapt to the devices where they are running [8]. Therefore, we developed the YanuX Orchestrator to gather information about the capabilities of the devices, i.e., displays, speakers, microphones, cameras, input devices, sensors and other details.

The YanuX Orchestrator is available for Android devices and Linux PCs. It runs in the background to continuously update the YanuX Broker because capabilities can change over time, e.g., a monitor can be connected to a laptop. These capabilities can then be taken into account by YanuX Coordinator distribution algorithm. Table 1 summarizes the information that can be gathered or inferred about the devices.

\section{CONCLUSIONS AND FUTURE WORK}

We have presented how the YanuX Framework provides useful abstractions and tools to allow developers to create applications that run across multiple co-located devices. We provide tools for the automatic distribution of the UI based on the capabilities of the devices running the applications, on restrictions defined by the developers, and on relationships established between the co-located devices. Notwithstanding, we allow users to easily override the distribution of the components according to their preferences. We have also enabled users to manage and share multiple application states (resources) to design environments where multiple users can interact and collaborate.

However, there are still more improvements that we wish to make. We will need to continue refining the YanuX Broker and YanuX Coordinator to better support a wider range of scenarios. In the future, we intend to expand the collaboration capabilities and to support multi-tasking involving multiple applications. We envision that the YanuX Orchestrator will listen to events sent from the YanuX Broker and react accordingly by managing applications, splitting the screen for simultaneous multitasking in public displays, or dealing with cross-device interaction gestures. We also intend to improve the positioning system and to take better advantage of its absolute positioning capabilities. For instance, a radar widget could be used to represent devices and the components currently
Table 1: Extracted Device Capabilities

\begin{tabular}{|c|c|}
\hline Name & Description \\
\hline Type & $\begin{array}{l}\text { String, e.g., desktop, laptop, tablet, smart- } \\
\text { phone or smart TV }\end{array}$ \\
\hline Display & Single Object or Array \\
\hline Type & String, e.g., internal, external or touchscreen \\
\hline Orientation & String, e.g., landscape or portrait \\
\hline Resolution & Array[Integer]/pixel, e.g., 1 to $\infty$ \\
\hline Bit depth & Integer/bit, e.g., 1 to $\infty$ \\
\hline Refresh Rate & Float/Hz, e.g., 0 to $\infty$ \\
\hline Size & Float/millimeter, e.g., 0 to $\infty$ \\
\hline Pixel Density & Integer/pixels per inch, e.g., 0 to $\infty$ \\
\hline Pixel Ratio & Float, e.g., 0 to $\infty$ \\
\hline Virtual Resolution & Array[Integer]/pixel, e.g., 1 to $\infty$ \\
\hline Speakers & Single Object or Array \\
\hline Type & String, e.g., loudspeaker or headphones \\
\hline Channels & Integer, e.g., 1 to $\infty$ \\
\hline Bit depth & Integer/bit, e.g., 1 to $\infty$ \\
\hline Sampling Rate & Float/Hz, e.g., 0 to $\infty$ \\
\hline Camera & Single Object or Array \\
\hline Type & $\begin{array}{l}\text { String, e.g., main, telephoto, wide-angle, } \\
\text { selfie or webcam }\end{array}$ \\
\hline Resolution & Array[Integer]/pixel, e.g., 1 to $\infty$ \\
\hline Bit Depth & Integer/bit, e.g., 1 to $\infty$ \\
\hline Refresh Rate & Float/Hz, e.g., 0 to $\infty$ \\
\hline Microphone & Single Object or Array \\
\hline Channels & Integer, e.g., 1 to $\infty$ \\
\hline Bit Depth & Integer/bit, e.g., 1 to $\infty$ \\
\hline Sampling Rate & Float/Hz, e.g., 0 to $\infty$ \\
\hline Input & $\begin{array}{l}\text { Array[String], e.g., keyboard, mouse, stylus, } \\
\text { touchscreen or speech input }\end{array}$ \\
\hline Sensors & $\begin{array}{l}\text { Array[String], e.g., GPS, accelerometer, gy- } \\
\text { roscope, compass, barometer, light or prox- } \\
\text { imity }\end{array}$ \\
\hline
\end{tabular}

associated with them, with redistribution of components being accomplished through drag-and-drop.

We intend to use YanuX in the development of applications that respond to societal challenges, besides continuing to develop prototype applications from which we have already received encouraging feedback $[31,32]$. In fact, we have recently concluded a user study supported by an application developed with the framework (see the following video: https://youtu.be/0q4qtyWWCpM). In addition to continuing to assess how people can benefit from applications running across multiple co-located devices, it is equally important to understand if developers can easily use our framework to build their own multi-device applications. Therefore, we also intend to conduct developer focused studies to evaluate the usability and acceptance of the framework from that point of view.

\section{ACKNOWLEDGMENTS}

This work is funded by Fundação para a Ciência e Tecnologia (FCT) through a Ph.D. Studentship grant (SFRH/BD/96899/2013). It is also 
supported by NOVA LINCS Research Center which is partially funded by project UID/CEC/04516/2020 granted by FCT.

\section{REFERENCES}

[1] J. Bradley and N. Agarwal. 2015. Proof Key for Code Exchange by OAuth Public Clients. Technical Report 7636. 1-20 pages. https://doi.org/10.17487/RFC7636

[2] Ramon F. Brena, Juan Pablo García-Vázquez, Carlos E. Galván-Tejada, David Muñoz-Rodriguez, Cesar Vargas-Rosales, and James Fangmeyer. 2017. Evolution of Indoor Positioning Technologies: A Survey. Fournal of Sensors 2017 (March 2017), 1-21. https://doi.org/10.1155/2017/2630413

[3] Pedro Centieiro, Rui Neves Madeira, Teresa Romão, A. Eduardo Dias, and Nuno Correia. 2015. In sync with fair play!: delivering a synchronized and cheatpreventing second screen gaming experience. In Proceedings of the 12th International Conference on Advances in Computer Entertainment Technology - ACE '15. ACM Press, New York, New York, USA, 1-11. https://doi.org/10.1145/2832932. 2832953

[4] Marco Cruz, Teresa Romão, Pedro Centieiro, and A. Eduardo Dias. 2017. Explor ing the use of second screen devices during live sports broadcasts to promote social interaction. In Proceedings of the 14th Conference on Advances in Computer Entertainment Technology - ACE '17, Vol. 10714 LNCS. Springer, Cham, 318-338. https://doi.org/10.1007/978-3-319-76270-8_23

[5] David Dearman and Jeffery S. Pierce. 2008. It's on my other computer!: computing with multiple devices. In Proceeding twenty-sixth Annu. CHI Conf. Hum. factors Comput. Syst. - CHI '08. ACM Press, New York, New York, USA, 767. https: //doi.org/10.1145/1357054.1357177

[6] Tao Dong, Elizabeth F. Churchill, and Jeffrey Nichols. 2016. Understanding the challenges of designing and developing multi-device experiences. In DIS 2016 Proc. 2016 ACM Conf. Des. Interact. Syst. Fuse. ACM Press, New York, New York, USA, 62-72. https://doi.org/10.1145/2901790.2901851

[7] Andrea Gallidabino and Cesare Pautasso. 2016. The Liquid.js Framework for Migrating and Cloning Stateful Web Components across Multiple Devices. In Proc. 25th Int. Conf. Companion World Wide Web - WWW'16 Companion. ACM Press, New York, New York, USA, 183-186. https://doi.org/10.1145/2872518.2890538

[8] Andrea Gallidabino, Cesare Pautasso, Ville Ilvonen, Tommi Mikkonen, Kari Systa, Jari-Pekka Voutilainen, and Antero Taivalsaari. 2016. On the Architecture of Liquid Software: Technology Alternatives and Design Space. In 2016 13th Work. IEEE/IFIP Conf. Softw. Archit. IEEE, 122-127. https://doi.org/10.1109/WICSA 2016.14

[9] Fabio Giglietto and Donatella Selva. 2014. Second Screen and Participation: A Content Analysis on a Full Season Dataset of Tweets. 7. Commun. 64, 2 (April 2014), 260-277. https://doi.org/10.1111/jcom.12085

[10] Saul Greenberg, Nicolai Marquardt, Till Ballendat, Rob Diaz-Marino, and Miaosen Wang. 2011. Proxemic interactions: the new ubicomp? interactions 18, 1 (Jan 2011), 42. https://doi.org/10.1145/1897239.1897250

[11] Edward Twitchell Hall. 1990. The Hidden Dimension (27 ed.). Anchor Books. 217 pages. https://books.google.com/books?id=HlmqAAAACAAJ\{\&\}pgis=1

[12] D. Hardt. 2012. The OAuth 2.0 Authorization Framework. Technical Report. https://doi.org/10.17487/rfc6749

[13] John Hartman, Udi Manber, and L Peterson. 1996. Liquid software: A new paradigm for networked systems. Technical Report. Tucson, AZ, USA.

[14] Tom Horak, Andreas Mathisen, Clemens N. Klokmose, Raimund Dachselt, and Niklas Elmqvist. 2019. Vistribute: Distributing Interactive Visualizations in Dynamic Multi-Device Setups. In Proc. 2019 CHI Conf. Hum. Factors Comput. Syst. - CHI '19. ACM Press, New York, New York, USA, 1-13. https://doi.org/10.1145/ 3290605.3300846

[15] Kantar TNS Germany. 2019. The Connected Consumer. https://www.google $\mathrm{com} /$ publicdata/explore?ds=dg8d1eetcqsb1

[16] Nicolai Marquardt, Till Ballendat, Sebastian Boring, Saul Greenberg, and Ken Hinckley. 2012. Gradual engagement: facilitating information exchange between digital devices as a function of proximity. In Proc. 2012 ACM Int. Conf. Interact. tabletops surfaces - ITS '12. ACM Press, New York, New York, USA, 31. https: //doi.org/10.1145/2396636.2396642

[17] Nicolai Marquardt, Robert Diaz-Marino, Sebastian Boring, and Saul Greenberg. 2011. The Proximity Toolkit: Prototyping Proxemic Interactions in Ubiquitous Computing Ecologies. In Proc. 24th Annu. ACM Symp. User interface Softw. Technol. - UIST '11. ACM Press, New York, New York, USA, 315. https://doi.org/10.1145/ 2047196.2047238

[18] Nicolai Marquardt, Ken Hinckley, and Saul Greenberg. 2012. Cross-device interaction via micro-mobility and f-formations. In Proc. 25th Annu. ACM Symp. User interface Softw. Technol. - UIST '12. ACM Press, New York, New York, USA, 13 https://doi.org/10.1145/2380116.2380121

[19] Tommi Mikkonen, Kari Systä, and Cesare Pautasso. 2015. Towards Liquid Web Applications. In 15th Int. Conf. Web Eng. ICWE 2015, Vol. 9114. Springer, Cham, Rotterdam, the Netherlands, 134-143. https://doi.org/10.1007/978-3-319-198903_10
[20] Partha Mukherjee and Bernard J. Jansen. 2015. Correlation of Brand Mentions in Social Media and Web Searching Before and After Real Life Events: Phase Analysis of Social Media and Search Data for Super Bowl 2015 Commercials. In 2015 IEEE Int Conf. Data Min. Work. IEEE, 21-26. https://doi.org/10.1109/ICDMW.2015.60

[21] Timothy Neate, Matt Jones, and Michael Evans. 2017. Cross-device media: a review of second screening and multi-device television. Pers. Ubiquitous Comput. 21, 2 (April 2017), 391-405. https://doi.org/10.1007/s00779-017-1016-2

[22] Michael Nebeling. 2017. XDBrowser 2.0: Semi-Automatic Generation of CrossDevice Interfaces. In Proceedings of the 2017 CHI Conference on Human Factors in Computing Systems - CHI '17. ACM Press, New York, New York, USA, 4574-4584. https://doi.org/10.1145/3025453.3025547

[23] Katie O'Leary, Tao Dong, Julia Katherine Haines, Michael Gilbert, Elizabeth F. Churchill, and Jeffrey Nichols. 2017. The Moving Context Kit: Designing for context shifts in multi-device experiences. In DIS 2017 - Proc. 2017 ACM Conf. Des. Interact. Syst. ACM, New York, NY, USA, 309-320. https://doi.org/10.1145/ 3064663.3064768

[24] F. Orujov, R. Maskeliūnas, R. Damaševičius, Wei Wei, and Ye Li. 2018. Smartphone based intelligent indoor positioning using fuzzy logic. Future Generation Computer Systems 89 (dec 2018), 335-348. https://doi.org/10.1016/j.future.2018.06.030

[25] Tim Paek, Maneesh Agrawala, Sumit Basu, Steve Drucker, Trausti Kristjansson, Ron Logan, Kentaro Toyama, and Andy Wilson. 2004. Toward universal mobile interaction for shared displays. In Proc. 2004 ACM Conf. Comput. Support. Coop. Work - CSCW'04. ACM Press, New York, New York, USA, 266. https://doi.org/ $10.1145 / 1031607.1031649$

[26] Seonwook Park, Christoph Gebhardt, Roman Rädle, Anna Feit, Hana Vrzakova, Niraj Dayama, Hui-Shyong Yeo, Clemens Klokmose, Aaron Quigley, Antti Oulasvirta, and Otmar Hilliges. 2018. AdaM: Adapting Multi-User Interfaces for Collaborative Environments in Real-Time. In Proc. 2018 CHI Conf. Hum. Factors Comput. Syst. - CHI '18. ACM Press, New York, New York, USA, 1-14. https://doi.org/10.1145/3173574.3173758 arXiv:1803.01166

[27] Fabio Paternò. 2019. Concepts and design space for a better understanding of multi-device user interfaces. Univers. Access Inf. Soc. 19, 2 (June 2019), 409-432. https://doi.org/10.1007/s10209-019-00650-5

[28] Fabio Paternò and Carmen Santoro. 2012. A logical framework for multi-device user interfaces. In Proc. 4th ACM SIGCHI Symp. Eng. Interact. Comput. Syst. - EICS '12 (EICS '12). ACM Press, New York, New York, USA, 45. https://doi.org/10. $1145 / 2305484.2305494$

[29] Nat Sakimura, John Bradley, Michael B. Jones, Breno de Medeiros, and Chuck Mortimore. 2014. OpenID Connect Core 1.0 incorporating errata set 1. Technical Report. http://openid.net/specs/openid-connect-core-1 0.html

[30] Pedro Albuquerque Santos, Rui Neves Madeira, and Nuno Correia. 2013. FCT4U - When Private Mobile Displays Meet Public Situated Displays to Enhance the User Experience. In 2013 IEEE 10th Int. Conf. Ubiquitous Intell. Comput. 2013 IEEE 10th Int. Conf. Auton. Trust. Comput. IEEE, 186-193. https://doi.org/10.1109/UICATC.2013.112

[31] Pedro Albuquerque Santos, Rui Neves Madeira, and Nuno Correia. 2018. Designing a Framework to Support the Development of Smart Cross-device Applications. In Proc. 17th Int. Conf. Mob. Ubiquitous Multimed. - MUM 2018. ACM Press, New York, New York, USA, 367-374. https://doi.org/10.1145/3282894.3289727

[32] Pedro Albuquerque Santos, Rui Neves Madeira, and Nuno Correia. 2019. YanuX Pervasive distribution of the user interface by co-located devices. In Proc. 16th EAI Int. Conf. Mob. Ubiquitous Syst. Comput. Netw. Serv. ACM, New York, NY, USA, 368-377. https://doi.org/10.1145/3360774.3360832

[33] Stephanie Santosa and Daniel Wigdor. 2013. A field study of multi-device workflows in distributed workspaces. In Proc. 2013 ACM Int. 7t. Conf. Pervasive ubiquitous Comput. - UbiComp '13. ACM Press, New York, New York, USA, 63. https://doi.org/10.1145/2493432.2493476

[34] Henrik Sørensen, Dimitrios Raptis, Jesper Kjeldskov, and Mikael B Skov. 2014. The 4C framework: Principles ofinteraction in digitalecosystems. In UbiComp 2014 Proc. 2014 ACM Int. Jt. Conf. Pervasive Ubiquitous Comput. (UbiComp '14). ACM Press, New York, New York, USA, 87-97. https://doi.org/10.1145/2632048.2636089

[35] Antero Taivalsaari, Tommi Mikkonen, and Kari Systa. 2014. Liquid Software Manifesto: The Era of Multiple Device Ownership and Its Implications for Software Architecture. In 2014 IEEE 38th Annu. Comput. Softw. Appl. Conf. IEEE, 338-343. https://doi.org/10.1109/COMPSAC.2014.56

[36] David Thevenin and Joëlle Coutaz. 1999. Plasticity of User Interfaces: Framework and Research Agenda. In Human-Computer Interact. INTERACT '99 IFIP TC. 13, M. Angela Sasse and Chris Johnson (Eds.). IOS Press, Amsterdam, The Netherlands, 110-116. http://iihm.imag.fr/publs/1999/interact99\{ \}plasticite.pdf

[37] WAMP. 2021. The Web Application Messaging Protocol - Web Application Messaging Protocol version 2 documentation. https:/wamp-proto.org/ 